Glosse

\title{
Ist der Herzkatheter ein Placebo?
}

Wir alle kennen das: Es gibt Nachrichten, mit denen wir es so halten möchten wie Queen Victoria, die sehr alt wurde, obwohl ihr die Segnungen der modernen Kardiologie - sprich der Herzkatheter - noch nicht zur Verfügung standen. Als die besagte Königin mit der Evolutionstheorie von Charles Darwin konfrontiert wurde, dass der Mensch vom Affen abstammt, soll sie diesen unglaublichen Angriff der Wissenschaft auf ihr Menschenbild mit einem Blick gen Himmel seufzend folgendermaßen kommentiert haben: Dann wollen wir zunächst einmal hoffen, dass es nicht stimmt, und wenn es doch stimmen sollte, so wollen wir alles dafür tun, dass es niemand erfährt.

Eine vergleichbare Gemütsbewegung durchlief die Kardiologenschaft, als sie auf dem TCT-Kongress mit den Ergebnissen der ORBITA-Studie konfrontiert wurde. Diese war als Geburtstagsgeschenk geplant: exakt vor 40 Jahren wurde die Ballondilatation aus der Taufe erhoben. Doch das ist ganz schön in die Hose gegangen! Eigentlich möchte keiner über diese Studie reden. Und Herr Grüntzig dürfte sich im Grab rumdrehen.

Doch worum ging es in dieser brisanten Studie? Ein englischer Kardiologe erdreistete sich, eine Studie aufzulegen, bei der die perkutane Katheterintervention (PCI) bei Patienten mit stabiler koronare Herzerkrankung (KHK) mit einer Scheinprozedur verglichen wurde. Und in der Tat, man mag es nicht glauben und kaum aussprechen, im Hinblick auf die Symptomminderung ergab sich kein Unterschied.

Provokativ könnte man sagen: Die Herzkatheter-Therapie ist bei stabiler KHK nicht wirksamer als Placebo. Diese Studie kratzt gewaltig am Selbstverständnis und auch am Selbstwertgefühl der interventionell tätigen Kardiologen. Tag für Tag rackern sie sich im Katheterlabor ab und attackieren jede Stenose mit der festen Überzeugung, etwas Gutes zu tun. Und jetzt das! Da bleibt zunächst nur das victorianische Stoßgebet. Doch arbeitslos werden sie ja nicht; denn auch ein Scheineingriff ist ein Eingriff, und den muss ja auch irgendjemand durchführen.

signifikanter Unterschied zwischen echter PCI und PCI-Simulation festgestellt werden. Das Ergebnis hat in kurzer Zeit u. a. in den sozialen Medien einen Sturm an Reaktionen ausgelöst.

In der zeitgleich im Fachblatt „The Lancet“ publizierten Studie sind an fünf Zentren in Großbritannien 200 KHK-Patienten mit koronarer 1-Gefäß-Erkrankung (Stenosegrad > 70\%) nach Randomisierung in verblindeter Form einer PCI oder einer Placebo-Prozedur unterzogen worden. In den sechs Wochen vor dem Eingriff waren alle Patienten bei sehr intensiver und in der Praxis so nicht üblicher Betreuung auf eine optimierte antianginöse Medikation eingestellt worden.

\section{Kein Unterschied bei der Belastungsfähigkeit}

Sechs Wochen nach dem Eingriff wurde die Wirkung im Belastungstest ermittelt. In beiden Gruppen war in Relation zum Eingangstest eine Zunahme der Belastungszeit zu verzeichnen, die nur in der PCI-Gruppe signifikant war $(28,4$ vs.
Dr. med. Peter Stiefelhagen

gunsten der PCI erwies sich als nicht signifikant $(p=0,200)$. Auf Basis einer "konservativen“ Studienplanung sollte ein Unterschied um 30 Sekunden aufgedeckt werden.

Dass dies nicht gelungen ist, liegt sicher nicht daran, dass es sich um triviale Koronarstenosen gehandelt hat. Messungen der prozentualen Stenosefläche und hämodynamischen Relevanz der Stenosen (FFR-Messung) ergaben vielmehr, dass anatomisch und funktionell de facto schwere Koronarverengungen vorlagen. Auch wurde nach der Intervention per FFR-Bestimmung dokumentiert, dass sich die koronare Hämodynamik deutlich verbessert hatte. Stressecho-Untersuchungen lieferten zudem Hinweise auf eine Abnahme von Ischämien. Auf die Symptome wirkte sich all dies gleichwohl nicht entscheidend aus.

\section{Müssen die Leitlinien geändert werden?}

Ist die PCI bei stabiler KHK damit als reine Placebo-Therapie überführt? Und müsste diese dann in den Leitlinien bezüglich ihres Stellenwerts abgewertet werden? Darüber wird es in nächster Zeit mit Sicherheit eine heftige und wohl auch emotional geprägte Kontroverse geben. ORBITA-Studienleiterin Dr. Rasha AlLamee aus London schien die Bedeutung der Studie eher herunterspielen zu wollen: Nach ihrer Einschätzung würde eine

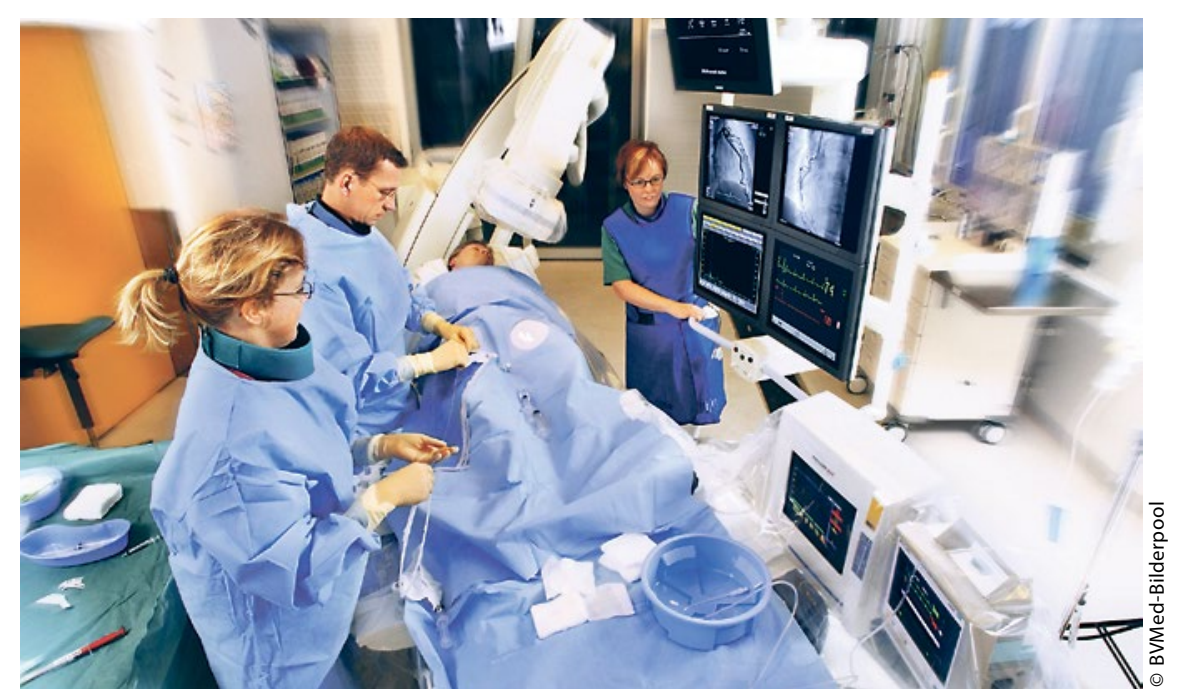

Wird im Katheterlabor künftig weniger los sein? Die Ergebnisse der ORBITA-Studie sprechen jedenfalls dafür, den Stellenwert der PCI bei stabiler KHK herabzustufen. 